\title{
1 Modelling Bluetooth Inquiry for SUMO
}

Michael Behrisch, Gaby Gurczik

Institute of Transportation Systems, German Aerospace Center, Rutherfordstraße 2, 12489 Berlin, Germany

\{Michael.Behrisch, Gaby.Gurczik\}@DLR.de

\subsection{Abstract}

SUMO provides an interface for the implementation of arbitrary additional vehicle devices. This paper describes how this interface was used to implement Bluetooth devices with a special focus on the inquiry process and how its modelling relates to real world measurements and a simple analytic model.

Keywords: Traffic simulation, Bluetooth, Inquiry Modelling

\subsection{Introduction}

Bluetooth [5] is a short-range, low-power, IEEE open standard for implementing wireless personal area networks. Bluetooth operates in the globally unlicensed $2.4 \mathrm{GHz}$ short-range radio frequency spectrum. Since there is a potential problem of interference from other devices using this frequency band, Bluetooth uses a Frequency-Hopping Spread Spectrum (FHSS) scheme, where devices alternate rapidly among the 79 available frequencies to transmit data. To set up an actual connection to exchange the necessary information between two Bluetooth devices, the so called inquiry process is designed to scan for other devices within range and thereby to discover each other. During the inquiry (discovery) process, one Bluetooth device (the master) enters the inquiry substate, whereas the other Bluetooth device (the slave) enters the inquiry scan substate. In the inquiry process the 48-bits unique MAC address and the internal clock-offset are exchanged in order to set up a lasting connection [5, $3,8]$.

Bluetooth devices are available in a number of vehicles and depict an easy way of detecting motions of persons and goods. Since every device is uniquely identifiable via its MAC address the devices can also be used to redetect vehicles over long ranges giving way to new applications of traffic monitoring. Since every Bluetooth device can be detected the data is ubiquitously available from headsets and navigational devices and also from in vehicle detectors such as tire pressure measurements. It is also easy to equip small devices such as smartphones to act as a detector making Bluetooth a universally accessible data source. 


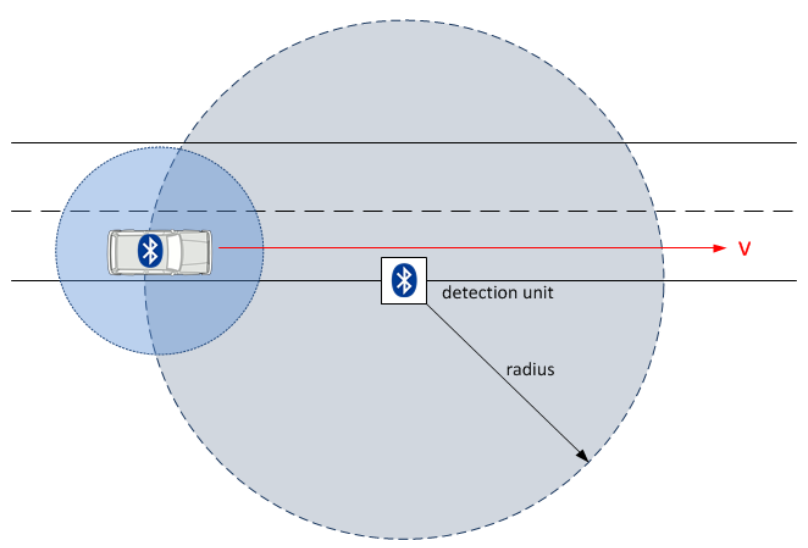

Figure 1 The Bluetooth detection principle

The German Aerospace Center (DLR) developed a traffic monitoring approach, called DYNAMIC $[1,7]$, which combines the advantages of Floating Car Data (FCD) and Floating Observer Data (FOD) principles. DYNAMIC is based on detections which are made by floating traffic observers using wireless radio-based technologies such as Bluetooth while passing other traffic objects (vehicles, cyclists, pedestrians). For the evaluation of the performance of DYNAMIC it is crucial to know how likely it is that a detectable traffic object (i.e. with Bluetooth device on board) within the detection range will be monitored. The major point to answer this question is the inquiry process which sets up the connection between Bluetooth devices and which can take up to several seconds. Given the possibly high speed of the vehicles and the relatively small detection range this poses a major problem to this detection mechanism. This paper focusses on a simple model for the inquiry process, describes its outcomings and the implementation of the process in the Bluetooth model of SUMO $[2,6]$ and compares it to real world measurements. The first section will focus on the analytical part, the second will describe the implementation in SUMO and the scenario used for evaluation and finally we will compare the theoretical and the simulative results with real world measurements.

\subsection{State of the Art}

In this paper we deal with the modeling of the inquiry process performance due to integrate the model in SUMO so that we can simulate Bluetooth detection behavior for stationary as well as mobile Bluetooth traffic monitoring systems. Since empirical analyses are complex and costly, a benchmarking implement is of particular importance. Unfortunately, researches in terms of evaluating Bluetooth traffic monitoring take Bluetooth performance mostly for granted. Therefore, they consider only frame conditions like distance between detector location and street, detection range and vehicle speed [10,12]. The inquiry process and with it the Bluetooth technology in itself is no object of research.

Thus, we had a closer look at related works from special field computer engineering where several researches deal with formal analysis or empirical approaches to model the inquiry process performance. For example in [4] a formal analysis using probabilistic model checking is developed to compute the expected time required for a master device to successfully receive replies from listening slave devices. On the basis of two different empirical approaches (first model using observation windows, second model using FHS interval times), [3] try to find 
out whether the number of inquirer and inquiry scanners has an effect on the discovery time. In [8] a detailed analysis of the interaction between Bluetooth devices in the inquiry and inquiry scan substates is given to analytically derive the inquiry time probability density function. Nevertheless, they state that precise inquiry time characterization is difficult due to the complex temporal and spectral interactions between two devices (for details see [8, 9, 11]).

Difficulties in using these models occur since that work is in the majority of cases older research of the time when Bluetooth was introduced as short-range communication technology between electronic devices. Therefore, these researches typically refer to Bluetooth specification version 1.1 which is important to know since the main difference in terms of the protocol is that, in version 1.1, the receiver only sends replies to every second message received. Hence, a device has to be discovered twice before it is actually considered to be discovered [4]. Furthermore, most researches are based on the assumption of an ideal, error-free environment, where messages never get lost [8]. This is, especially in our special field of studies, not lifelike.

For this reason, we investigate a simple model for the inquiry process in this paper, which fulfils our purpose while at the same time considering the specific behavior of the Bluetooth inquiry process.

\subsection{Analytical Modelling of the Inquiry}

The inquiry will be modelled as a frequency scanning process which lets the detector determine the frequency the vehicle device is using for the communication. Since the detector may change the order in which it scans for every pass and the device may change its frequency as well and we have no a priori knowledge about the distribution we assume every frequency and every order is equally likely.

The real inquiry process as described in [5] is much more complicated, it involves two trains of frequencies which change after every scan while the device to be detected only shows up in regular intervals. We will make use of these properties in our modelling later on.

Assuming a length of the scanning interval of $l$ then the target frequency is one point in each of the successive intervals. The task is now to calculate the probability that another interval of length $t$ (modelling the travel time in the detection range) contains at least one of the points.

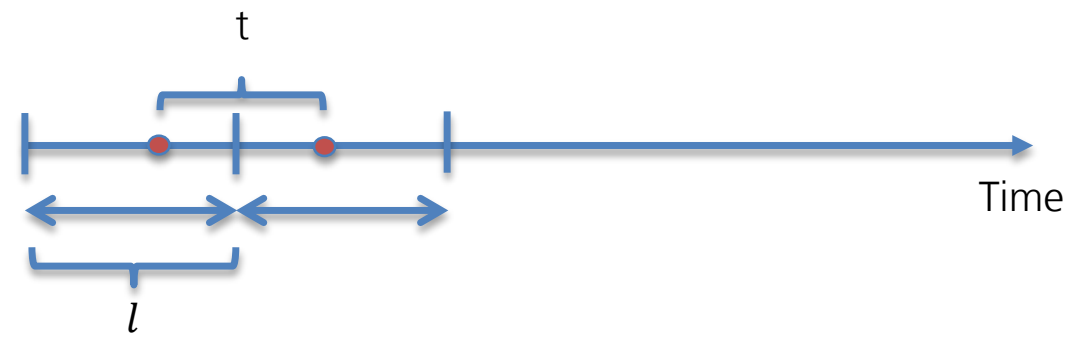

Figure 2 Model of the inquiry process with two scanning intervals of length I

We distinguish three cases depending on the relation of travel time and scanning interval: 
1. $t<l$

2. $l \leq t<2 l$

3. $t \geq 2 l$ : The detection probability is obviously 1

We solve case 1 and 2 by integrating over the position of the starting point of the travel interval in the first scanning interval and then dividing by the length of the interval. The integration always needs to be split into the cases where $t$ lies completely in $l$ and where $t$ can be divided into a part a in I and a part outside $l$ :

$t<l$ :

$$
\begin{aligned}
P_{2}(t, l)=\frac{\int_{0}^{l} p(t, l, x) d x}{l} & \\
= & \frac{1}{l} \int_{0}^{l-t} \frac{t}{l} d x+\frac{1}{l} \int_{l-t}^{l} 1-\left(1-\frac{l-x}{l}\right)\left(1-\frac{t-(l-x)}{l}\right) d x \\
= & \frac{t}{l}-\frac{t^{3}}{6 l^{3}}
\end{aligned}
$$

$l \leq t<2 l$ :

$$
\begin{aligned}
P_{2}(t, l)=\frac{\int_{0}^{l} p(t, l, x) d x}{l} & \\
= & \frac{1}{l} \int_{0}^{2 l-t} 1-\left(1-\frac{l-x}{l}\right)\left(1-\frac{t-(l-x)}{l}\right) d x+\frac{1}{l} \int_{2 l-t}^{l} 1 d x \\
= & 1-\frac{(2 l-t)^{3}}{6 l^{3}}
\end{aligned}
$$

The resulting function depicting the probability depending on the travel time ratio is shown in Figure 3. For l we can assume the length of the scanning interval which is about 2.56s.

During the evaluation of the theoretical result we found a simpler exponential model to fit the data even better. The major drawback of the first approach is that the detection is assumed to be for sure if the interval is larger than $2 \mathrm{l}$, so there is no possibility of a miss right after this point. To handle this case more gracefully and also get closer to the real world functions presented below, we assume that we have a fixed detection probability $\mathrm{p}_{\mathrm{d}}$ whenever the detector happens to be online simultaneously with the device to be detected. We assume this probability to be close to 0.5 , because the detector as described above may be in the wrong train when the device appears and so it may scan the wrong frequencies. On the other hand the device is long enough online that it is principle possible that (provided the train is correct) every frequency is detected. The number of tries for a detection is calculated by the ratio of the travel time $t$ and the interval between two online events $b$ of the device (which is about 0.64s). We assume that there are on average $\mathrm{t} / \mathrm{b}$ detection tries, so the simple resulting formula is:

$$
P_{1}\left(t, p_{d}, b\right)=1-\left(1-p_{d}\right)^{\frac{t}{b}}
$$

A third function was taken into account when evaluating the first practical results which also resembles the fact that the detector might need an additional amount of time to recover after each detection and thus may take a longer period before detecting all of a number of 
available devices. The function is of a similar general shape as the binomial form above, but to get a better fit an additional exponent was introduced. Fitting to the data resulted in:

$$
P_{3}(t)=1-e^{-.24 * t^{2.68}}
$$

A comparison of the three function shows the picture below

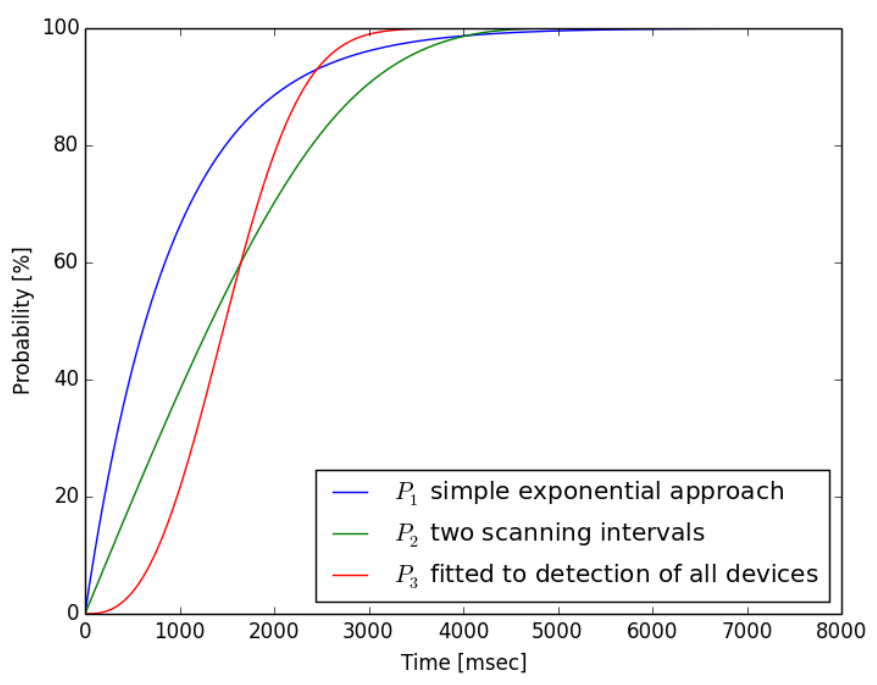

Figure 3 Comparison of modelling functions

\subsection{The Simulation Implementation}

To evaluate our analytical results SUMO was extended by the functionality to specify whether a traffic object works as Bluetooth transmitter (BTsender) or Bluetooth receiver (BTreceiver). BTsender are all the vehicles which can be detected by the BTreceivers. In practice that means that these vehicles have a Bluetooth device on board. Furthermore they have no additional functionality. The vehicles which are defined to be BTreceivers are our Floating Traffic Observers which are used for traffic monitoring. Every simulated vehicle can be a BTsender or a BTreceiver, it can also have both properties or none tof them (i.e. being a simple traffic object with no additional Bluetooth features). To control the Bluetooth detection in SUMO global parameters like equipment rates for BTsender and/or BTreceiver or the detection range can be stated using the command line options. The mentioned functionalities where implemented for SUMO version 0.19.0.

The implemented detection process in SUMO calculates the time the BTsender is in the detection range of the BTreceiver and determines the probability whether a detection took place purely based on this time. The first implementation also available in SUMO 0.19.0 used the function $P_{3}$ above but was found to have two major drawbacks compared to real world data as well as analytical evaluation: The relatively slow incline at the start and later increase of the first derivative in the process. There is no delay to be expected in the detection of the first device so the new detections should become less and less in the course of the process as it happens with $P_{1}$ and $P_{2}$.

When choosing between $P_{1}$ and $P_{2}$ there is (beside the property of not being fixed to 1 after a certain amount of time mentioned above) an additional benefit of $\mathrm{P}_{1}$ related to the implementation. Since the simulation determines in every simulation step anew whether a detection took place, the probabilities should be additive, that is, it should be easy to calculate the probability that there was a detection in the joined interval $t_{1}+t_{2}$ from the 
individual probabilities that there were detections either in $t_{1}$ or $t_{2}$. As it turns out this can be easily achieved with the exponential distribution above.

$$
\begin{aligned}
P_{1}\left(t_{1}+t_{2}, p_{d}, b\right)=1- & \left(1-p_{d}\right)^{\frac{t_{1}+t_{2}}{b}}=1-\left(1-p_{d}\right)^{\frac{t_{1}}{b}}\left(1-p_{d}\right)^{\frac{t_{2}}{b}} \\
& =1-\left(1-P_{1}\left(t_{1}, p_{d}, b\right)\right)\left(1-P_{1}\left(t_{2}, p_{d}, b\right)\right)
\end{aligned}
$$

Where the last term denotes exactly the probability of two independent throws in successive intervals. This combination of probabilities is not possible with the other approaches.

\subsection{The Simulation Scenario}

The underlying network for our simulation scenario is a representation of the DLR test track, the Ernst-Ruska-Ufer (abbreviated ERU in the figures below) in Berlin-Adlershof. It includes a total track length of about $4 \mathrm{~km}$ with one major road $(1.4 \mathrm{~km}$ with two directions) and several incoming and outgoing minor roads. We simulated a whole day with the demand and the route chpoices being calculated directly from induction loop data for the 11.1.2011.

There is a total demand of about 30000 vehicles including about $4 \%$ trucks and busses. In a first step the scenario was calibrated to the detector data such that network effects as a major traffic jam in the late afternoon on the eastbound direction are correctly reflected in the simulation.

The Bluetooth related parameters are the following:

- $\quad$ one fixed BTreceiver in each direction (see the green spots)

- $\quad$ fixed BTsender equipement rate of $30 \%$

- detection range $100 m$

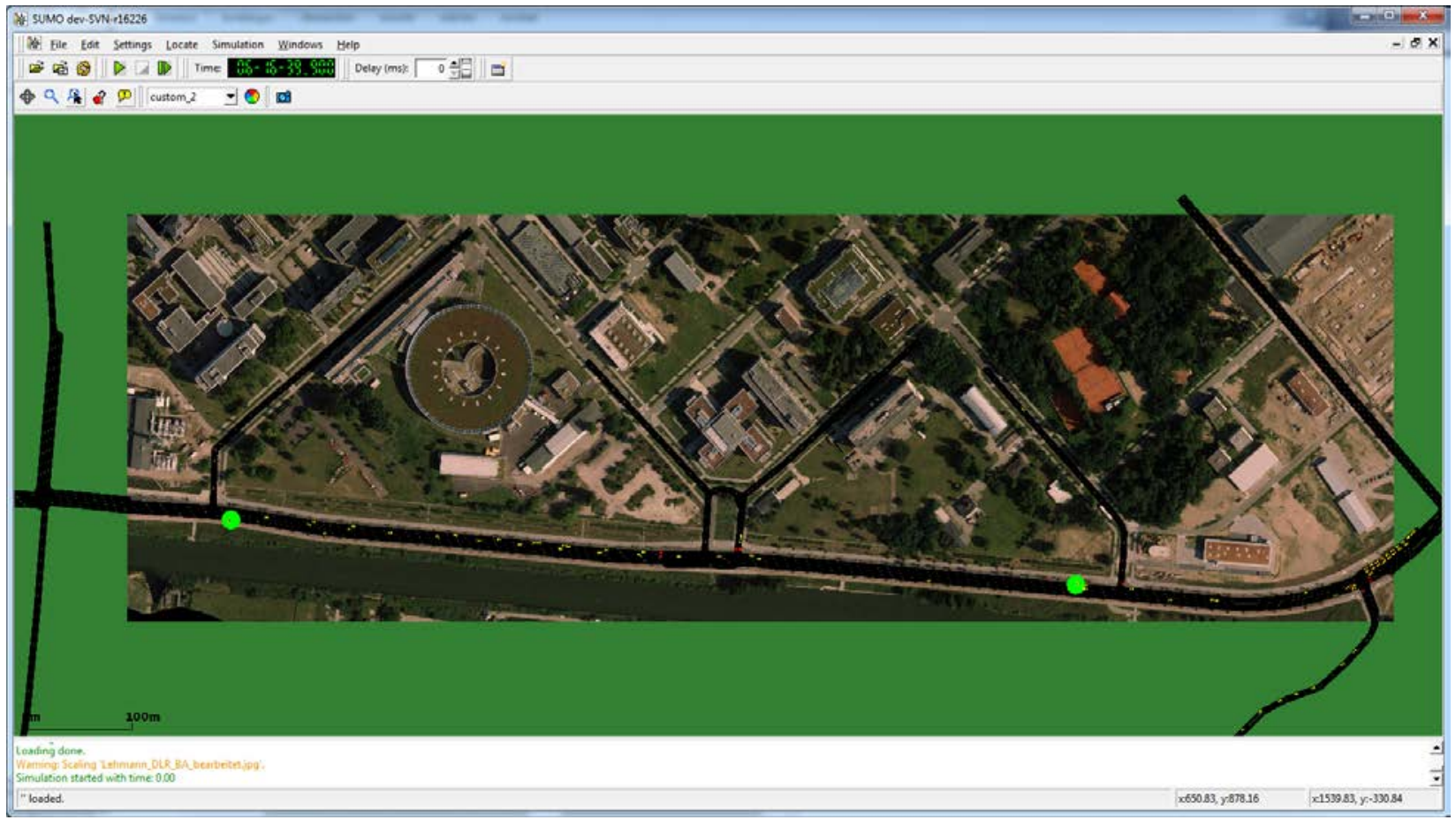

Figure 4 Test track scenario in the final SUMO simulation (green points denote the bluetooth detectors) 


\subsection{Comparison to Real World Measurements}

In order to derive the exponential function mentioned above, laboratory as well as field experiments with Bluetooth receivers and senders were conducted to measure detection rates as a function of inquiry times.

In the laboratory test 1 (respectively 2) BTreceivers were stationary installed to find 1, 2, 4 or 6 BTsender within the detection range. The BTsenders were transmitting their signal continuously, whereas the BTreceiver(s) were periodically restarted after 10 seconds of being in inquiry mode. Every time, one of the BTsenders was detected, a data set including timestamp, BTsender-ID and signal strength value was stored to a log file.

Figure 5 illustrates the results from the laboratory test. For the varying number of BTreceivers and/or BTsenders the probability density is given. There you can see that more than $80 \%$ of all detections are realised within a time interval of 1 second (1000 milliseconds). For the probability density we looked at the intertimes. The intertimes are the time differences between a detection of a BTsender and the starting time of the inquiry mode of the BTreceiver respectively a previous detection time of that specific BTsender. That means the intertimes are exactly that times it took a BTreceiver to detect a BTsender provided it is in the detection range. In the laboratory test nearly $100 \%$ of all detectable devices were detected after 3 seconds independent of the number of BTreceivers and BTsenders.

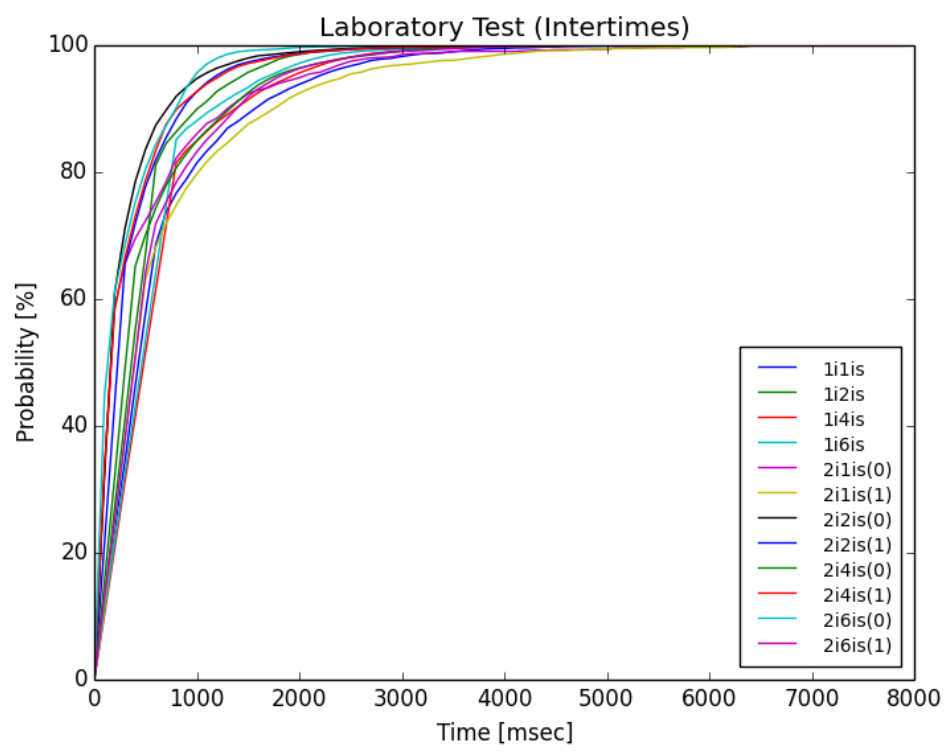

Figure 5 Probabiity density for the laboratory tests

In the laboratory test almost perfect conditions were given for the BTreceiver to detect BTsenders. The reality looks somewhat different - besides several error sources (e.g. Bluetooth signal reflections or shading effects) the to be detected BTsenders are moving objects which makes detection less likely since the BTsenders are not permanently within detection range. Furthermore, the speed factor reduces the time the BTsender is within detection range additionally.

To evaluate how the inquiry time process is influenced from real environment a two-hour field test which took place on August 20th 2013 between 6 a.m. and 8 a.m. was conducted. In that field test 4 BTreceiver objects (observer) in form of cars moving along the street ErnstRuska-Ufer (two lanes per direction; approximately $1.4 \mathrm{~km}$ ) on the so called WISTA area in Berlin-Adlershof were used (see Figure). Contemporaneously, these objects were considered as BTsender (i.e. the traffic participants), which should be detected by the other observers. Within the observer cars our prototyped Bluetooth monitoring systems (called Bluetooth-Box, 
shortened "BluB") was installed. The observers moved freely according to their desired speed respectively to local feasibility and under consideration of the German Road Traffic Act (StVO).

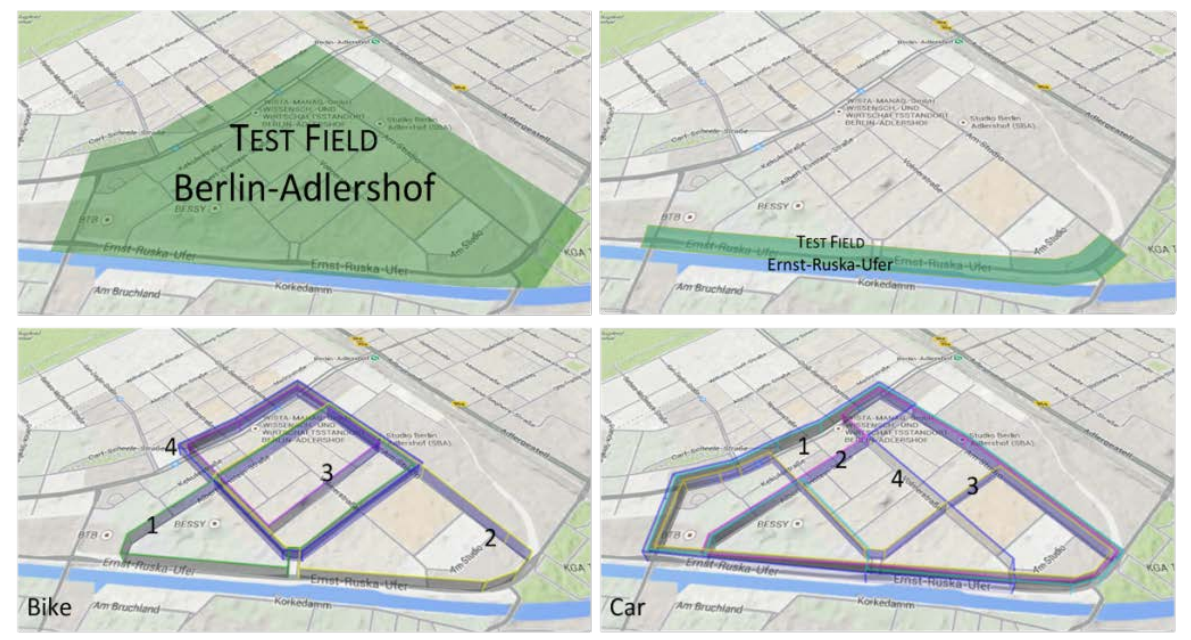

Figure 6 Defined field test observer routes (map source: Google): Ernst-Ruska-Ufer (upper right), car and cyclist routes (lower right and left) in second field test

The Ernst-Ruska-Ufer is both at once, public place and our DLR test track where additionally traffic monitoring infrastructure is installed to observe real-time traffic situations. Therefore, we could benefit from reference data collected from stationary Bluetooth detectors so that during the two hours two different types of data were collected. On the one hand, we monitored the traffic via stationary Bluetooth detectors. On the other hand, a mobile detection was done by our four moving observer vehicles. For both data types, the same data sets as in the laboratory test were stored containing timestamp, BTsender-ID and signal strength value.

The results of the stationary Bluetooth measurements are given in Figure 7. The left figure shows the results from the specific field test day (August 20th 2013). For higher reliability we permanently installed our BluBs at two points of the Ernst-Ruska-Ufer for several months so that we could benefit from long-term measurements. The right figure shows the results from the long-term measurements. It is obvious that the probability density is quite similar. Due to still undefined explicit error values the increase is less sharp in comparison to the laboratory data. Especially between 1 and 6 seconds the course of the function is smoother than that under laboratory conditions. Still unclear are effects where no inclination is observable within longer time slots (e.g. from $1500 \mathrm{~ms}$ to $5000 \mathrm{~ms}$ in the left figure and from $2000 \mathrm{~ms}$ to nearly $4000 \mathrm{~ms}$ in the right figure). It would mean that there were no inquiry times which are that long. Since there are even longer time periods in the data it might be of methodical reason.
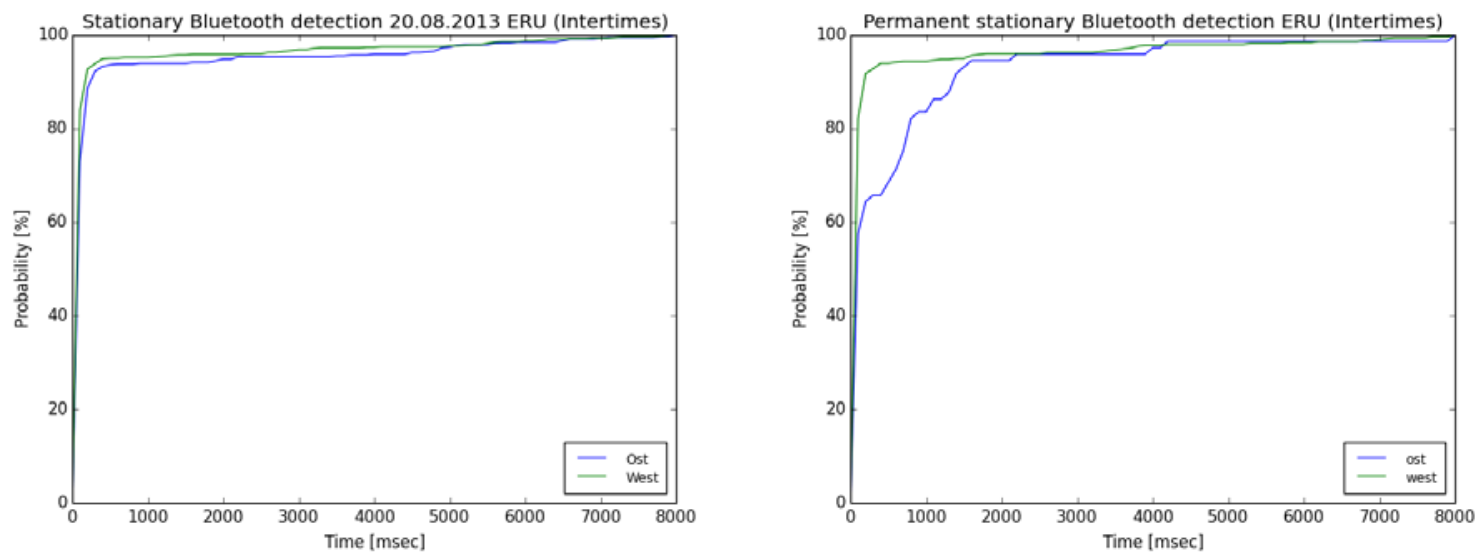

Figure 7: Probability density for field test results on Ernst-Ruska-Ufer (left: two-hour test on August 20 2013 ; right: permanent stationary Bluetooth detection) 
The results collected from moving Bluetooth observer vehicles are illustrated in Figure 8 . The course of the functions is similar to that of the stationary Bluetooth measurements for all four observers even if that of observer car 1 seems to be more consistent. A reason might be the amount of collected data which was the biggest from car 1. Interesting is that the same effect of time slots without inclination is observable in that case as well. It seems to occur always between approximately $2000 \mathrm{~ms}$ and $7000 \mathrm{~ms}$.

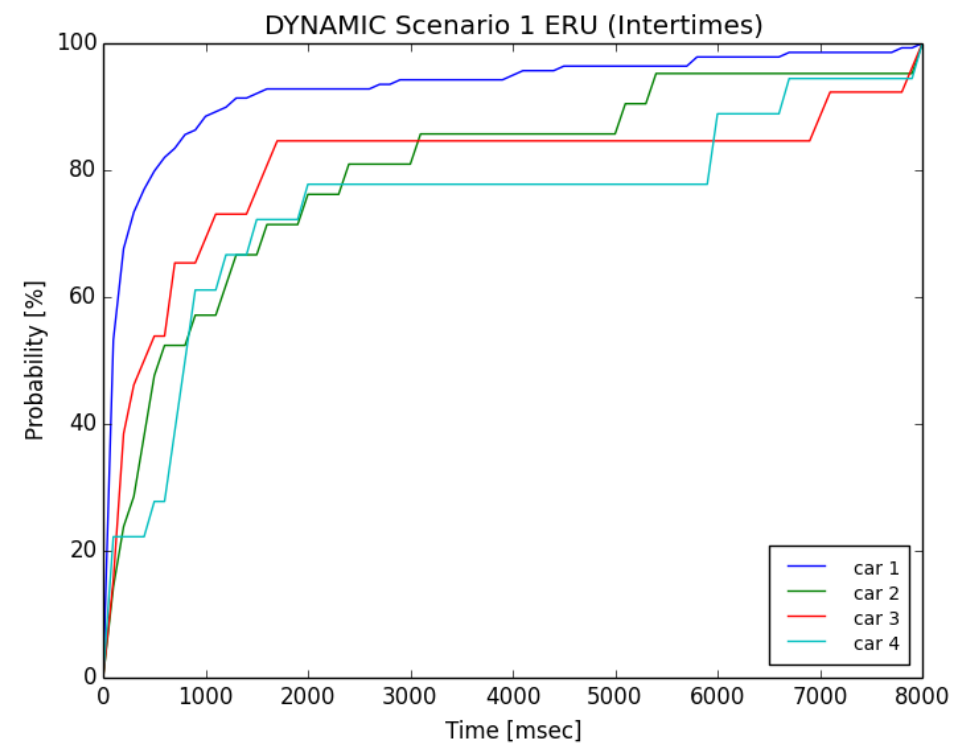

Figure 8: Probability density for field test results on Ernst-Ruska-Ufer using moving observer (cars)

In addition to the field test on Ernst-Ruska-Ufer, several test runs with 8 moving observers using multimodal BTreceiver objects (i.e. cars and cyclists) were conducted on other routes on the WISTA area (Figure 6) to see whether the results affirm our conclusion. These additional field tests took even place on August 20th 2013, but from 9 a.m. to 10 a.m. and 1 p.m. to 3 p.m. Note that in these field tests observer car 3 (red line) had some major problems in collecting data. Nevertheless the results from these area wide measurements show better accordance with the results derived from laboratory tests.
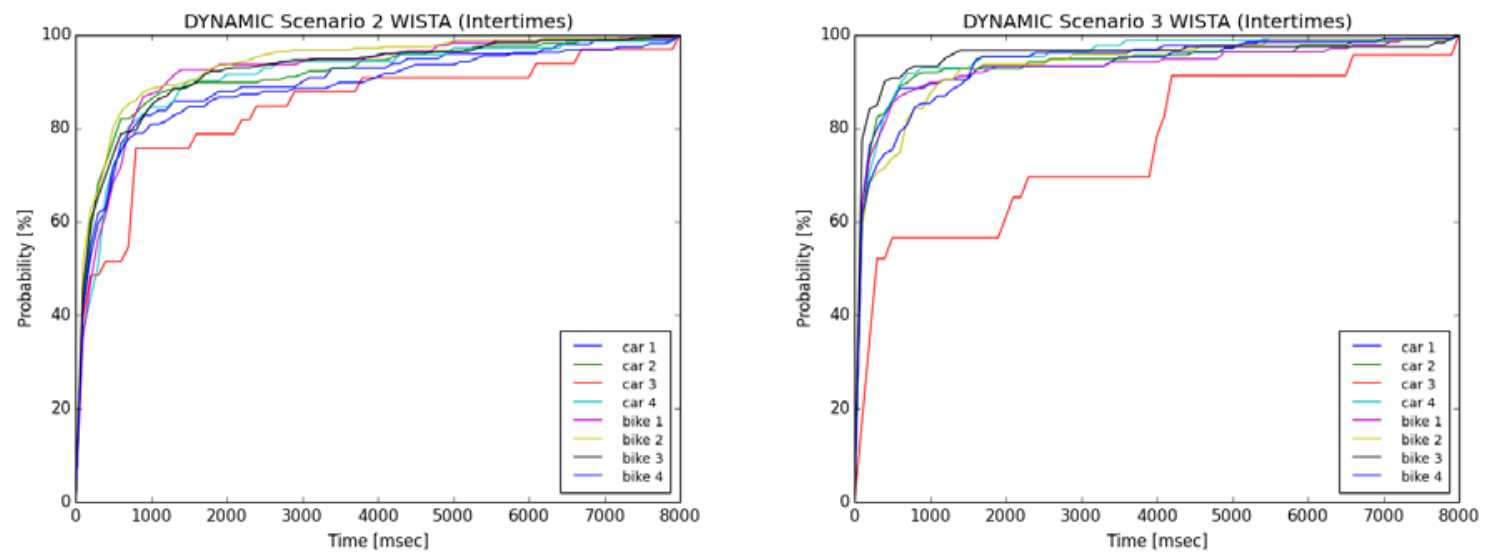

Figure 9: Probability density for additional field test results on WISTA area using cars and cyclists as moving observers

Figure 10 shows the results from the simulation scenario. For both simulated stationary Bluetooth detection units (modelling the East and West measuring bridges on the ErnstRuska-Ufer), the probability density to detect vehicles with Bluetooth devices on board within a specific time interval (in seconds) is given. One can see that in more than $80 \%$ the equipped traffic objects are discovered in a time interval less than one second. The results differ 
between the two monitoring positions. That is possibly due to the jam occurring in the eastern part of the scenario which leads to far more (re-)detections of waiting vehicles in shorter time intervals. All in all, the density probabilities look very similar especially to the results from the real world stationary Bluetooth measurements (see Figure 7, cf. curve 'ERU_ost' and 'ERU_west' in the right figure).
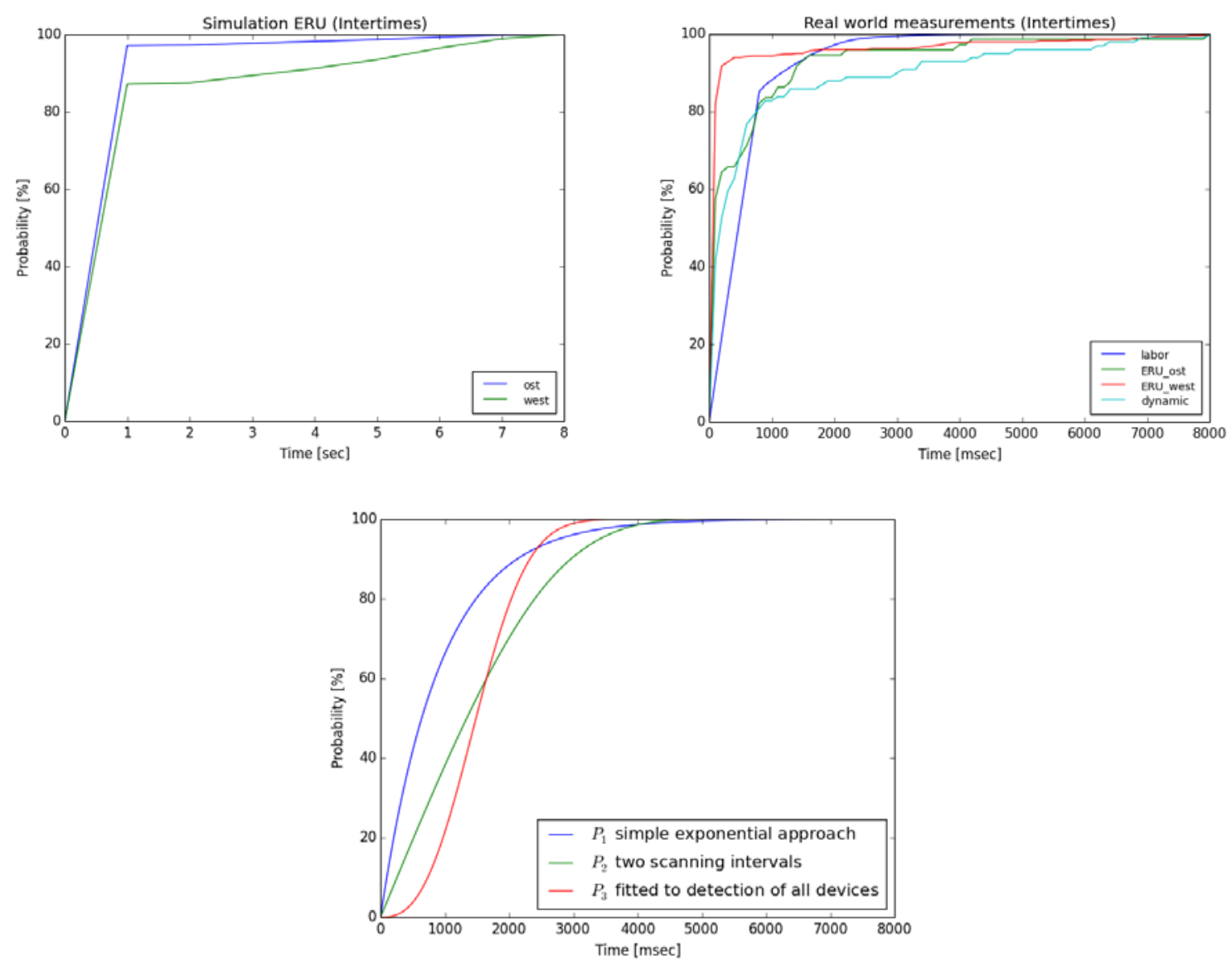

Figure 10: Probability density for simulation results (top left) compared to a summary of most important results from real world measurements (top right) and the theoretical results (bottom)

What we learn from this comparison is that the probability density seems to be best fitted by a exponential distribution which makes sense since the number of detections based on Bluetooth is a sequence of $n$ independent seen/not seen trials each of which occurs with probability $\mathrm{p}$. This follows from the assumption that the number of vehicles equipped with Bluetooth devices and the number of observer vehicles within the network is small, so that the chances to encounter are statistically independent events. Therefore, the existences of those encounter respectively detection events can be described using a exponential distribution.

\subsection{Conclusions and Discussions}

To sum up the following results can be stated from the experiments:

- The probability density seems to be best fitted by a exponential distribution.

- Within 1 second more than $90 \%$ of all detections are done under laboratory condition; in real environment conditions at least $80 \%$. That means most of the detectable BTsenders in detection range are found within the first second. 
- In case of moving observers field test results show better accordance with laboratory results than the stationary Bluetooth measurements. It has to be kept in mind that laboratory results reflect perfection.

- Simulation results fit the stationary Bluetooth monitoring results quite well.

- The simulations carried out are in good agreement with the empirical data as well as the theoretical model.

One weakness of our approach is that we can not detect the inquiry time directly but can only detect the interval between two successful inquiries, so in the case of small traffic densities we will need different measurements to validate our data. This will be a subject to further research. Additionally we need to investigate further the unusual plateau behavior in the dynamic cases (see Figure 8) where we often had no additional detections between second 2 and second 7 .

\section{Acknowledgements}

We thank Daniel Krajzewicz for the initial implementation of the Bluetooth detection mechanism in SUMO and Andreas Luber for performing the laboratory experiments and providing their data.

\section{References}

[1] Ruppe, S., Junghans, M., Haberjahn, M., Troppenz, C.: Augmenting the Floating Car Data Approach by Dynamic Indirect Traffic Detection. In Proceedings of Transport Research Arena - Europe 2012, Athens, Greece (2012)

[2] Krajzewicz, D., Erdmann, J., Behrisch, M., Bieker, L.: Recent Development and Applications of SUMO - Simulation of Urban MObility. International Journal On Advances in Systems and Measurements, 5 (3\&4):128-138 (2012)

[3] Franssens, A.: Impact of multiple inquirers on the Bluetooth discovery process - And its application to localization. University of Twente (2010)

[4] Duflot, M., Kwiatkowska, M., Norman, G., Parker, D.: A Formal Analysis of Bluetooth Device Discovery. International Journal on Software Tools for Technology Transfer, Volume 8, Issue 6, pp 621-632 (2006)

[5] Specification of the Bluetooth system, version 4.0, Bluetooth SIG, 2010, http://www.bluetooth.com.

[6] SUMO - Simulation of Urban Mobility, available at http://sumo-sim.org/

[7] Gurczik, G., Junghans, M., Ruppe, S.: Conceptual Approach for Determining Penetration Rates for Dynamic Indirect Traffic Detection. ITS World Congress 2012, Vienna, Austria (2012)

[8] Peterson, B.S., Baldwin, R.O., Kharoufeh, J.P.: Bluetooth Inquiry Time Characterization and Selection. In IEEE Transactions on mobile computing, Volume 5, Issue 9 (2006)

[9] Peterson, B.S., Baldwin, R.O., Kharoufeh, J.P.: A Specification-Compatible Bluetooth Inquiry Simplification. In Proceedings of the 37th Hawaii International Conference on System Sciences (HICSS'04), Waikoloa, Hawaii (2004) 
[10] Hoyer R., Leitzke C.: Verfahrenstechnische Bedingungen für die Reisezeitbestimmung mittels Bluetooth-Technologie. In Proceedings of Heureka 2011, Kassel, Germany (2011)

[11] Kasten, O., Langheinrich, M.: First Experiences with Bluetooth in the Smart-ITS Distributed Sensor Network. In Proceedings of 2001 International Conference on Parallel Architectures and Compilation Techniques (PACT'01), Barcelona, Spain (2001)

[12] Wasson J. S., Sturdevant J. R., Bullock D.M.: Real-Time Travel Time Estimates Using Media Access Control Address Matching. Institute of Transportation Engineers Journal (ITE Journal), Volume 78, Issue 6, pp 20-23, June (2008) 